\title{
Space Studies of the Black-Drop Effect at a Mercury Transit
}

\author{
Glenn Schneider \\ Steward Observatory, 933 N. Cherry Ave., University of Arizona, \\ Tucson, Arizona 85721
}

Jay M. Pasachoff

Williams College-Hopkins Observatory, 33 Lab Campus Dr., Williamstown, MA 01267

Leon Golub

Smithsonian Astrophysical Observatory, Mail Stop 58, 60 Garden St., Cambridge, MA 02138

\begin{abstract}
Transits of Mercury and Venus across the face of the Sun are rare. The 20th century had 15 transits of Mercury and the 21st century will have 14, the two most recent occurring on 15 November 1999 and 7 May 2003. We report on our observations and analysis of a black-drop effect at the 1999 and 2003 transits of Mercury seen in high spatial resolution optical imaging with NASA's Transition Region and Coronal Explorer (TRACE) spacecraft. We have separated the primary contributors to this effect, solar limb darkening and broadening due to the instrumental point spread function, for the 1999 event. The observations are important for understanding historical observations of transits of Venus, which in the 18 th and 19 th centuries were basic for the determination of the scale of the solar system. Our observations are in preparation for the 8 June 2004 transit of Venus, the first to occur since 1882 . Only five transits of Venus have ever been seen - in $1639,1761,1769,1874$, and 1882 . These events occur in pairs, whose members are separated by 8 years, with an interval between pairs of 105 or 122 years. Nobody alive has ever seen a transit of Venus.
\end{abstract}

\section{Motivation}

Historically, transits of Venus were the major method for hundreds of years of determining the Astronomical Unit and thus the scale of the solar system, given that Kepler's laws of 1609/1618 are mere proportions. Edmond Halley presented a method of determining the A.U. by observing the durations of the chords across the Sun from a number of different locations on Earth. Accordingly, dozens of expeditions from many countries traveled around the world for the 18th and 19th-century transits, most famously including the voyage of Captain James Cook, who was sent to Tahiti to observe the 1769 event.

The accuracy of the measurements was severely impaired, however, by the "black-drop effect," in which the silhouette of Venus did not separate cleanly from the limb of the Sun during its inner contact. The timing accuracy was thus closer to a minute than to the expected second or two. As Schaefer (2001) 
has shown, many people have mistakenly attributed, and continue to mistakenly attribute, the black-drop effect to the atmosphere of Venus.

Our space observations of the transit of Mercury have shown the presence of a black-drop effect (Figure 1). Since Mercury has no substantial atmosphere and since the observations were taken from outside the Earth's atmosphere, clearly the effect - at least for Mercury - has causes other than due to a planetary atmosphere. By implication, Venus's black-drop effect would arise, at least in part, from similar causes.

\section{Observations}

The Transition Region and Explorer spacecraft can observe the Sun in a variety of ultraviolet wavelengths. For our studies of the 1999 transit of Mercury, we used only data from its broad band white-light channel. TRACE's 0.5 arc sec pixels, and temporally stable point-spread function unaffected by the Earth's atmosphere, give it spatial high resolution. A black-drop effect was observed in all frames near the point of internal tangency of the Mercurian and solar disks.

We discuss our calibrations, methods of analysis and extensive modeling in Schneider, Pasachoff \& Golub (2001, 2004). We found major contributors giving rise to the black-drop effect come from two causes, that of the pointspread function of the telescope and that of the solar limb darkening. Removing those two contributions left us with images of the limb of Mercury unaffected by the solar limb.

The data for the 1999 event were sent to Earth in lossless uncompressed image format. Though we had worked with the TRACE planning team for the 2003 event to obtain a white-light data set at higher temporal cadence, those observations were downlinked in a compressed format normally used for solar observations. A irrecoverable loss of image fidelity at the bottom end of the dynamic sampling range resulted, and we were unable to recover sufficient accuracy to repeat our earlier analysis. We will make sure that the data for the upcoming Venus transit are returned to Earth in uncompressed format.

\section{Future Work}

Our immediate intention is to observe the 8 June 2004 transit of Venus from TRACE in orbit and from telescopes on the ground. Massive world-wide efforts will take place to observe this Venus transit. The European Southern Observatory, for example, is coordinating a public observation campaign. The International Astronomical Union's Commission on Education and Development has a Web site at http://www.transitofvenus.info that lists past and future observations, shows images, and provides links. We also intend to observe the transit of Mercury of 8 November 2006. After that, the following transits of Mercury aren't until 9 May 2016 and 11 November 2019.

Acknowledgments. This work was partially supported by NSF grant ATM000545; Pasachoff's work on eclipses was also supported in part by NGS/CRE. TRACE is supported by a NASA/GSFC contract to the Lockheed Martin Corp. NICMOS IDT S/W (NASA grant NAG 5-3042) was used extensively. 

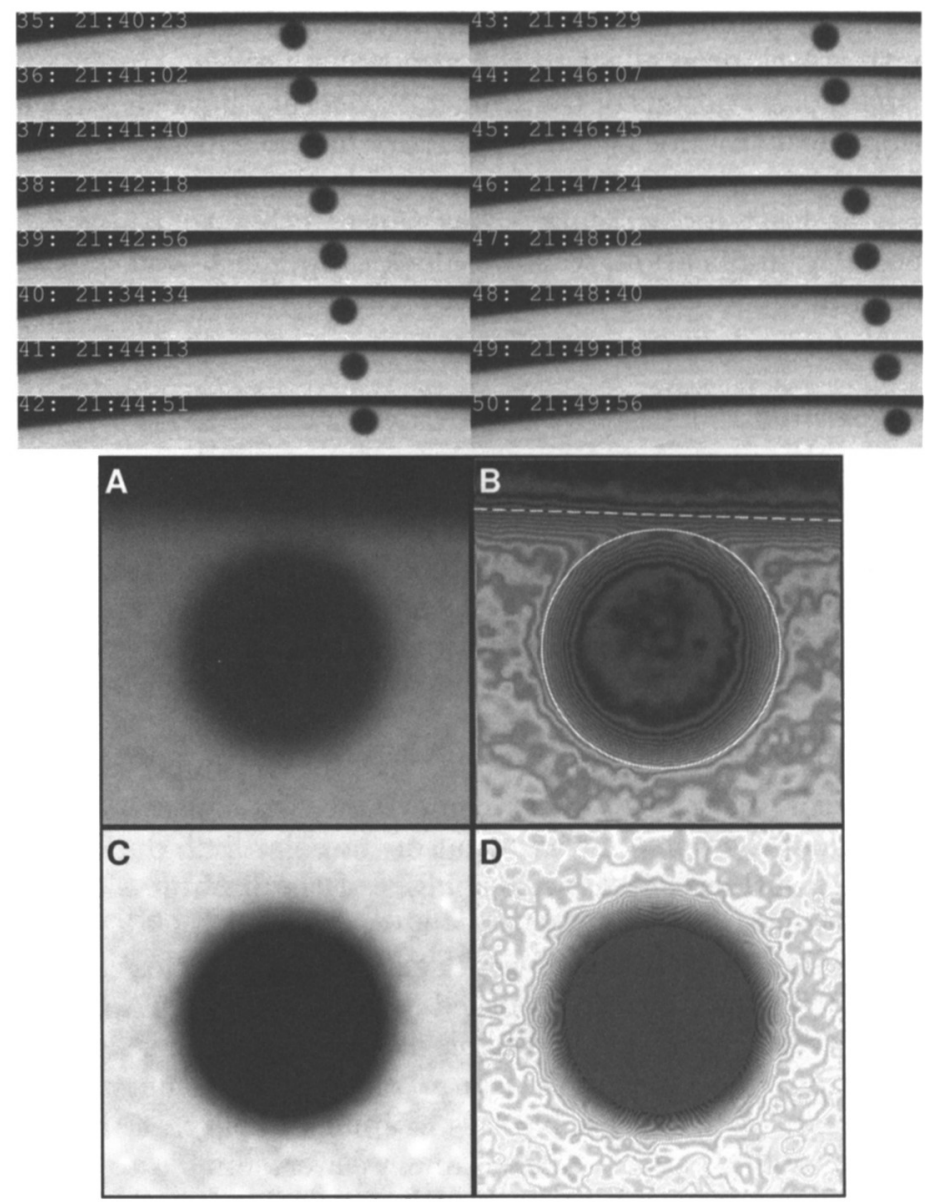

Figure 1. TRACE images. A-D from frame 50. A (image) \& B (5\% intensity contours; dashed line $=$ solar limb, white circle $=$ Mercury disk $)$. Post-processed: C \& D (1/2\% contours) show no black-drop.

\section{References}

Schaefer, B. E., 2001, JHA, xxxii, 325

Schneider, G., Pasachoff, J. M., \& Golub, L., 2001, BAAS, 33, 1037.

Schneider, G., Pasachoff, J. M., \& Golub, L., 2004, Icarus, in press. 